\title{
25 Research Square \\ Vestibular Nerve Function In Patients With Type 2 Diabetes Detected By Vestibular Evoked Myogenic Potentials
}

\section{Jinying Zhang}

Second Affiliated Hospital of Fujian Medical University

Jiayu Lin

Second Affiliated Hospital of Fujian Medical University

Huapin Huang ( $\nabla$ zhangjinying1989@163.com )

Fujian Medical University Union Hospital

\section{Research Article}

Keywords: Diabetes, Diabetic peripheral neuropathy, vestibular evoked myogenic potentials, Vestibular.

Posted Date: February 21st, 2022

DOI: https://doi.org/10.21203/rs.3.rs-1296202/v1

License: (c) This work is licensed under a Creative Commons Attribution 4.0 International License.

Read Full License 


\section{Abstract}

Introduction: Vestibular dysfunction may be present in type 2 diabetes mellitus. This relationship was frequently observed in diabetic peripheral neuropathy. The present study aimed to evaluate the vestibular function of diabetic patients and compare the results with those of a healthy adult control group.

Materials and Methods: Patients were asked to complete demographic characteristics form. Moreover, lipids, fasting plasma glucose, as well as hemoglobin A1C tests, were carried out on them. Both the patients and control group were evaluated using two vestibular tests including ocular Vestibular Evoked Myogenic Potential (o-VEMP), and cervical Vestibular Evoked Myogenic Potential (c-VEMP).

Results: The The o-VEMP and c-VEMP showed a significant difference in latencies between the four groups. OVEMP latency $\mathrm{p} 15$ and cVEMP latency $(\mathrm{p} 13, \mathrm{n} 23)$ were positively correlated with HbA1c, FPG, and illness duration, and oVEMP latency $\mathrm{n} 10$ was positively correlated with $\mathrm{HbA} 1 \mathrm{c}$ and $\mathrm{FPG}$, with statistically significant differences.

Conclusion: Based on the obtained results, vestibular dysfunctions are related to Diabetes. Patients with type two diabetes mellitus showed vestibular dysfunctions compared to the healthy control group.

\section{Introduction}

Vestibular evoked myogenic potential (VEMP) is an objective method for evaluating the otolithic apparatus and vestibular nerve conduction pathways in the vestibular system, such as the balloon and ellipsoid bursa, and is a myogenic potential recorded after stimulation of the otolithic apparatus by highintensity acoustic signals. ${ }^{[1]}$ Depending on the placement of the recording electrodes, VEMP examinations can be divided into cervical vestibular evoked myogenic potential (cVEMP) and ocular vestibular evoked myogenic potential (oVEMP). The oVEMP reflects the function of the superior vestibular pathway of the ellipsoid sac and the cVEMP reflects the function of the inferior vestibular pathway of the balloon.

Type 2 diabetes is a metabolic disorder characterized by chronic hyperglycemia caused by multiple etiologies. ${ }^{[2]}$ In China, the proportion of people over 20 years of age with diabetes is reported to be $9.7 \%$ (about 92.4 million people), and the incidence of abnormal glucose tolerance is $15.5 \%$ (148.2 million people). ${ }^{[3]}$ The increasing prevalence of diabetes and diabetic complications have become a major health problem for Chinese nationals and have imposed a heavy economic burden on society. Diabetes can cause a variety of clinical symptoms, among which balance dysfunction (dizziness, falls, etc.) is one of the symptoms frequently complained by patients with this disease. Recent epidemiological studies have shown that the rate of vestibular impairment in diabetic patients reaches $70 \%,{ }^{[4]}$ vestibular impairment may also be a major factor in diabetic balance disorders.

With the growing recognition of the impact of vestibular impairment on imbalance and falling in diabetic patients, the detection of diabetic vestibular function has also received attention. Diabetic patients may develop vestibular dysfunction in the absence of significant vestibular symptoms, which has recently 
been described as "subclinical vestibular neuropathy"..$[$ In such cases, vestibular lesions can be identified by objective vestibular diagnostic testing tools, despite the absence of vestibular symptoms in patients with DM. Previous studies have used electronystagmography instruments to assess changes in vestibular function using spontaneous nystagmus tests, position tests, head shake tests, neck turn tests, temperature tests, and visual oculomotor system examinations (gaze test, sweep test, smooth tracking test) ${ }^{[6]}$ but there are limitations due to the examination technique and patient cooperation. At present, VEMP testing constitutes an important part of the vestibular test battery and provides either diagnostic or assistive contributions in the clinical evaluation of common vestibular diseases such as Meniere's disease (MD), vestibular neuritis (VN) and superior canal dehiscence syndrome (SCDS) ${ }^{[7]}$ In this study, we assessed the vestibular function of diabetic patients by VEMP, analyzed the characteristics of their vestibular function changes, and investigated the relationship between diabetes and vestibular function impairment.

\section{Materials And Methods}

All procedures performed in studies involving human participants were in accordance with the ethical standards of the insti[1]tutional and/or national research committee and with the 1964 Helsinki declaration and its later amendments or comparable ethical standards.

\subsection{Participants}

Eighty-nine patients with type 2 diabetes who visited the Second Hospital of Fujian Medical University from April 2021 to November 2021 were enrolled in this study, including 57 males and 32 females with a mean age of (53.0 \pm 11.9 years). There were 42 cases in the healthy control group, including 23 males and 19 females, with a mean age of (52.3 \pm 5.6$)$ years. (for details, see Table 1$)$.The diagnosis of diabetes was based on the American Diabetes Association (ADA) (2012) diabetes diagnosis criteria. ${ }^{[8]}$ The normal subjects had no existing/ historical records of any otological disorders, nor any historical or existing vestibular complaints (such as dizziness, vertigo, nausea). Patients presenting with a history of head-andneck injury with limitation of neck movements, a history of head-and-neck surgery, central nervous system disorders; those presenting with diseases involving external or middle ear, those presenting with a history of usage of drugs such as ototoxic drugs, antiepileptics, muscle relaxants; those who could not cooperatewere excluded from the study. The patients gave permission for all of the procedures involving clinical tests and data collection, with approval by the ethical committee of the Second Hospital of Fujian Medical University, China. General information including gender, age, body mass index, duration of diabetes, hemoglobin A1C (HbA1c), fasting plasma glucose(FPG), blood pressure, smoking history, triglycerides, total cholesterol, low-density lipoprotein cholesterol (LDL-C), and high-density lipoprotein cholesterol (HDL-C) were recorded for all participants.

\subsection{Test used}

1.2.1 Nerve conduction velocity examination 
All enrolled patients were examined by a neurologist specializing in nerve conduction velocity (Danish Keypoint electromyography evoked potentials). Diabetic peripheral neuropathy was diagnosed using the 2010 American Diabetes Association (ADA) diagnostic criteria, ${ }^{[9]}$ and the enrolled patients were divided into 29 patients in the no peripheral neuropathy group (NDPN group), 26 patients in the subclinical diabetic peripheral neuropathy group (SDPN group), and 34 patients in the confirmed diabetic peripheral neuropathy group (DDPN group) according to the above criteria.

\subsection{2 cVEMP test}

All participants underwent cVEMP testing. Patients were placed in supine position during examination. Two active electrodes were placed in the upper $1 / 3$ of the SCM on both sides and two reference electrodes were placed near the sternum or sternum to record the cVEMP, the forehead was used as the location of the ground electrode. During the recording, the subject was instructed to lift the head from the pillow so that the sternocleidomastoid muscle contracted and maintained a muscle tension greater than $50 \mathrm{uV}$. The SCM was activated by elevating the head $20^{\circ}-30^{\circ}$ during the test to keep it in a tonic contraction state for a certain period of time. VEMP was recorded under bilateral short-acoustic $3-\mathrm{Hz}$ stimulation conditions with an intensity of $132 \mathrm{~dB}$ SPL, a stimulation rate of 5 times/s, band-pass filtering of 10-300 Hz, and superimposed 50-100 times. ${ }^{[10]}$ The presence of a VEMP response was assessed, and the parameters included latencies $(\mathrm{ms})$ and amplitudes $(\mathrm{mV})$. cVEMP presence was determined when reproducible short-latency biphasic waveforms appeared at certain latencies. The latencies of P13 and N23 were measured. The typical cVEMP waveform was bidirectional, with the positive wave latency near $13 \mathrm{~ms}$, labeled $\mathrm{p} 13$, and the negative wave appearing near $23 \mathrm{~ms}$, labeled n23. The recorded EMG activity was given a correction, and finally the difference between the two myopotentials was used, which reflects the reflected potential originating from the balloon.Unreproducible or unrecognizable waveforms were identified as the absence of responses.

\subsection{3 oVEMP test}

All patients underwent oVEMP testing. Patients were seated, and subjects were instructed to gaze upward at a target point $60-70 \mathrm{~cm}$ in front of the eye at an angle of $25^{\circ}$ from their horizontal line of sight when acoustic stimulation was given, and to keep their gaze at this position throughout the test so that the extraocular muscles were in an optimal state of tension. The test was completed in a standard soundproof room with a $0.5 \mathrm{kHz}$ short tone burst, a rise and fall time of $1 \mathrm{~ms}$, a peak duration of $2 \mathrm{~ms}$, a stimulus intensity of $100 \mathrm{~dB} \mathrm{nHL}$, a recording window width of $50 \mathrm{~ms}$, a stimulus rate of 5.1 times/s, and a cumulative superposition of 300 times. The electrode was placed in the middle of the forehead, the recording electrode was placed about $1 \mathrm{~cm}$ below the midpoint of the inferior orbital rim on both sides, and the reference electrode was placed $2-3 \mathrm{~cm}$ from the recording electrode. oVEMP waveforms were recorded from the contralateral extraocular muscles after the sound was given on one side. The N10-P15 composite waveform is recorded as negative followed by positive, and the trough with an upward trend is marked as N10 about $10 \mathrm{~ms}$ after the sound is given, and the peak with a downward trend after N10 is marked as P15. After 3 times of repeated sound giving at the same intensity, the recorded waveform with 
good repeatability is considered to be the oVEMP response waveform. If N10 and P15 are not observed in the recorded waveform, or the waveform recorded at the same intensity for 3 times is not reproducible, the oVEMP waveform disappears. ${ }^{[11]}$

\section{Data Analysis}

The data were analyzed using SPSS software(Version 26). The quantitative results were presented with the mean and standard deviation. Data between groups were analyzed by one-way ANOVA, and multiple comparisons were performed by LSD method. The the elicited rates of o-VEMP and c-VEMP testing were compared in two groups by chi-square test. The Pearson's correlation coefficient was used to evaluate the possible relationships between quantitative data. A p-value less than 0.05 or 0.01 ( $P \llbracket 0.05, P \llbracket 0.01$ ) was considered statistically significant.

\section{Results}

3.1 Comparison of general information of the four groups

Information on the subjects from four groups is provided in Table 1. There was no significant difference between the groups in terms of gender, age and lipids $(P>0.05)$. Compared with the control group, BMI was at a higher level in the NDPN group and SDPN group, and the difference was statistically significant $(P<0.05)$. fasting glucose and glycosylated hemoglobin levels were higher in the NDPN group, SDPN group, and DDPN group than in the control group, and there was a statistically significant difference $(P<0.01)$. glycosylated hemoglobin $(P<0.01)$ and fasting glucose $(P<0.05)$ levels were higher than in the NDPN and SDPN groups, and the duration of diabetes was also longer than in the NDPN $(P<0.01)$ and SDPN groups $(P<0.05)$, with no significant differences between the NDPN and SDPN groups $(P>0.05)$.

Table 1 Comparison of baseline information of the four groups 


\begin{tabular}{|c|c|c|c|c|}
\hline & Control group & NDPN group & SDPN group & DPN group \\
\hline Number of cases $(\mathrm{M} / \mathrm{F})$ & $42 \rrbracket 23 / 19 \rrbracket$ & $29 \otimes 19 / 10 \rrbracket$ & $26 \bigotimes 15 / 11 \rrbracket$ & $34 \llbracket 23 / 11 \rrbracket$ \\
\hline Age (/year) & $52.3 \pm 5.6$ & $56.1 \pm 9.1$ & $51.3 \pm 12.8$ & $51.6 \pm 13.1$ \\
\hline $\mathrm{BMI}(\mathrm{kg} / \mathrm{m} 2)$ & $22.5 \pm 2.11$ & $24.2 \pm 3.5^{\#}$ & $24.6 \pm 2.8^{\#}$ & $23.1 \pm 3.1$ \\
\hline $\mathrm{TG} \triangle \mathrm{mmol} / \mathrm{I} \mathbb{\square}$ & $1.7 \pm 1.1$ & $1.9 \pm 1.3$ & $1.8 \pm 0.8$ & $1.8 \pm 1.5$ \\
\hline $\mathrm{HDL}-\mathrm{C} \rrbracket \mathrm{mmol} / \mathrm{I} \mathbb{\mathrm { N }}$ & $1.3 \pm 0.4$ & $1.1 \pm 0.3$ & $1.2 \pm 0.1$ & $1.3 \pm 0.5$ \\
\hline LDL-C $₫ \mathrm{mmol} / \mathrm{I} \bigotimes$ & $2.9 \pm 1.1$ & $3.0 \pm 1.2$ & $3.2 \pm 1.0$ & $3.4 \pm 1.1$ \\
\hline $\mathrm{TC} \rrbracket \mathrm{mmol} / \mathrm{I} \rrbracket$ & $4.6 \pm 1.4$ & $4.7 \pm 1.2$ & $4.7 \pm 1.0$ & $5.2 \pm 1.4$ \\
\hline UA(umol/L) & $300.1 \pm 88.2$ & $355.7 \pm 106.5$ & $308.9 \pm 64.4$ & $325.3 \pm 89.7$ \\
\hline $\mathrm{HbA} 1 \mathrm{c}(\%)$ & $5.6 \pm 0.4$ & $8.6 \pm 2.1^{\#}$ & $8.7 \pm 2.1^{\#}$ & $10.9 \pm 2.5^{\#^{\star \star}}$ \\
\hline $\mathrm{FPG}(\mathrm{mmol} / \mathrm{L})$ & $5.1 \pm 0.5$ & $8.7 \pm 3.2^{\#}$ & $8.9 \pm 3.0^{\#}$ & $10.9 \pm 4.9^{\#^{*}}$ \\
\hline Illness duration(/year) & - & $4.2 \pm 4.5^{\#}$ & $4.5 \pm 5.1^{\#}$ & $7.3 \pm 5.5^{\#^{*}}$ \\
\hline
\end{tabular}

$\mathrm{F}=$ Female $\mathrm{M}=$ Male; $\mathrm{BMI}=$ body mass index; $\mathrm{TG}=$ Triglyceride; $\mathrm{HDL}-\mathrm{C}=$ high-density lipoprotein; $\mathrm{LDL}-$ $\mathrm{C}=$ Low density lipoprotein; $\mathrm{TC}=$ Cholesterol; $\mathrm{UA}=$ Uric acid; $\mathrm{HbA1}=$ hemoglobin $\mathrm{A} 1 \mathrm{C} ; \mathrm{FPG}=$ fasting plasma glucose; compared to healthy control and NDPN groups, ${ }^{*} p<0.01$; compared to SDPN group, ${ }^{*} p<0.05$, ${ }^{\star *} \mathrm{p}<0.01$.

\subsection{Comparison of VEMP elicitation rates in subjects}

A total of 84 ears were used in the healthy control group, of which 80 ears elicited oVEMP, with an elicitation rate of $95.2 \%$; 82 ears elicited cVEMP, with an elicitation rate of $97.6 \%$. The diabetic group had 262 ears, of which 236 ears elicited oVEMP, with an elicitation rate of $90.1 \%$; 240 ears elicited cVEMP, with an elicitation rate of $91.6 \%$. By chi-square test, there was no statistically significant difference between the two groups in oVEMP and cVEMP elicitation rates (oVEMP: $\chi^{2}=1.54$; cVEMP: $\chi^{2}=2.70$, both P>0.05).

\subsection{Comparison of oVEMP and cVEMP between the four groups}

Regarding oVEMP, the bilateral $\mathrm{n} 10$ and p15 latencies were prolonged in the SDPN and DDPN groups compared with the healthy control and NDPN groups (all $P<0.01$ ), and there was no difference between the bilateral $\mathrm{n} 10$ and $\mathrm{p} 15$ latencies in the SDPN and DDPN groups $(P>0.05)$. Amplitude did not differ between the groups $(P>0.05)$. Regarding cVEMP, the bilateral $p 13$ and $n 23$ latencies were prolonged in the SDPN and DDPN groups compared with the healthy control and NDPN groups (all $P<0.01$ ), and there was no difference between the bilateral p13 and n23 latencies in the SDPN and DDPN groups $(P>0.05)$. Amplitudes were not significantly different between the groups, as shown in Table 2. 
Table 2 Parameters of elicited cVEMP and oVEMP in four groups.

\begin{tabular}{|lllll|}
\hline & Control group & NDPN group & SDPN group & DDPN group \\
\hline Ln10(ms) & $11.3 \pm 0.9$ & $10.9 \pm 0.9$ & $12.2 \pm 1.1^{\#}$ & $12.5 \pm 1.0^{\#}$ \\
\hline Lp15(ms) & $14.9 \pm 0.8$ & $15.1 \pm 1.4$ & $17.0 \pm 1.0^{\#}$ & $16.8 \pm 1.2^{\#}$ \\
\hline LoAmp(uV) & $1.8 \pm 1.0$ & $1.6 \pm 0.7$ & $1.8 \pm 1.2$ & $1.8 \pm 1.5$ \\
\hline Rn10(ms) & $11.1 \pm 0.8$ & $11.0 \pm 1.1$ & $12.2 \pm 1.3^{\#}$ & $12.5 \pm 1.0^{\#}$ \\
\hline Rp15(ms) & $15.1 \pm 1.0$ & $15.1 \pm 1.3$ & $16.7 \pm 1.5^{\#}$ & $16.7 \pm 1.2^{\#}$ \\
\hline RoAmp(uV) & $1.6 \pm 1.2$ & $1.7 \pm 1.1$ & $1.6 \pm 1.1$ & $1.5 \pm 1.0$ \\
\hline Lp13(ms) & $12.1 \pm 0.9$ & $12.3 \pm 0.8$ & $13.3 \pm 1.5^{\#}$ & $13.6 \pm 1.6^{\#}$ \\
\hline Ln23(ms) & $21.8 \pm 1.1$ & $21.8 \pm 1.1$ & $23.6 \pm 1.9^{\#}$ & $24.1 \pm 1.7^{\#}$ \\
\hline LcAmp(uV) & $114.1 \pm 58.5$ & $94.6 \pm 51.3$ & $90.9 \pm 62.3$ & $99.8 \pm 46.4$ \\
\hline Rp13(ms) & $12.1 \pm 0.8$ & $12.4 \pm 0.8$ & $13.3 \pm 1.5^{\#}$ & $13.7 \pm 1.5^{\#}$ \\
\hline Rn23(ms) & $21.8 \pm 1.4$ & $21.8 \pm 1.7$ & $23.7 \pm 2.1^{\#}$ & $24.2 \pm 1.6^{\#}$ \\
\hline RcAmp(uV) & $114.6 \pm 58.0$ & $82.2 \pm 47.0$ & $89.3 \pm 42.1$ & $99.0 \pm 45.0$ \\
\hline
\end{tabular}

$\mathrm{L}=\mathrm{Left}, \mathrm{R}=$ Right; $\mathrm{n} 10=\mathrm{n} 10$ latency, p15=p15 latency, p13=p13 latency, n23=n23 latency; o=ocular vestibular evoked myogenic potential; $c=$ cervical vestibular evoked myogenic potential; Amp=Amplitude.

3.4 Correlation of HbA1c, FPG, and Illness duration with oVEMP and cVEMP parameters

As shown in Table 3, oVEMP latency p15 and cVEMP latency $(\mathrm{p} 13, \mathrm{n} 23)$ were positively correlated with $\mathrm{HbA} 1 \mathrm{c}, \mathrm{FPG}$, and illness duration, and oVEMP latency $\mathrm{n} 10$ was positively correlated with $\mathrm{HbA} 1 \mathrm{c}$ and FPG, with statistically significant differences.

Table 3 Comparison of HbA1c, FPG, and illness duration with VEMP parameters 


\begin{tabular}{|llll|}
\hline & HbA1c & FPG & illness duration \\
\hline Ln10 & $0.279^{* *}$ & $0.311^{* *}$ & 0.125 \\
\hline Lp15 & $0.492^{* *}$ & $0.456^{* *}$ & $0.231^{* *}$ \\
\hline LoAmp & 0.102 & 0.048 & 0.009 \\
\hline Rn10 & $0.365^{* *}$ & $0.370^{* *}$ & $0.244^{* *}$ \\
\hline Rp15 & $0.358^{* *}$ & $0.369^{* *}$ & $0.242^{* *}$ \\
\hline RoAmp & 0.006 & 0.020 & 0.071 \\
\hline Lp13 & $0.201^{*}$ & $0.256^{* *}$ & $0.224^{*}$ \\
\hline Ln23 & $0.247^{* *}$ & $0.227^{*}$ & $0.381^{* *}$ \\
\hline LcAmp & -0.079 & -0.112 & -0.031 \\
\hline Rp13 & $0.242^{* *}$ & $0.267^{* *}$ & $0.245^{* *}$ \\
\hline Rn23 & $0.269^{* *}$ & $0.288^{* *}$ & $0.326^{* *}$ \\
\hline RcAmp & -0.083 & -0.167 & -0.069 \\
\hline
\end{tabular}

${ }^{*} \mathrm{P}<0.05$.

${ }^{* *} \mathrm{P}<0.01$.

\section{Discussion}

In this study, the vestibular function in type 2 diabetic patients was evaluated. The results suggested that the latency $(\mathrm{N} 10, \mathrm{P} 15, \mathrm{P} 13, \mathrm{~N} 23)$ was prolonged in patients with SDPN group and DDPN group, compared to the NDPN group and healthy control group. The latency was related to glycemic control and illness duration.

The maintenance of the body's balance posture requires the combined action of the vestibular organs, visual senses, and proprioception. ${ }^{[12]}$ The peripheral sensory information provided by the vestibular, visual and proprioceptive senses is centrally organized and integrated, followed by visual localization via the vestibulo-ocular reflex and postural regulation via the vestibulospinal reflex. The patient's ability to maintain balance is successively diminished when vestibular function is impaired. Diabetes mellitus may increase the risk of vestibular impairment. ${ }^{[13]}$ Current studies have also confirmed the impairment of vestibular function in diabetes in pathomorphological studies, physiological change studies, and clinical studies.Perez et al. ${ }^{[14]}$ demonstrated functional impairment of the vestibular portion of the inner ear in the metabolic state of diabetes using vestibular end-organ testing and a diet-induced type 2 diabetes animal 
model. The study concluded that microangiopathy is the main cause of abnormal vestibular function and may be related to the cochlear ganglion having a common nerve sheath adjacent to the vestibular ganglion. Microangiopathy leads to ischemia of the vestibular structures and alters fluid metabolism in the inner ear, causing symptoms of vestibular hypofunction such as tinnitus, hearing loss, and vertigo in diabetic patients. ${ }^{[15]}$ In a pathomorphological study, Myers et al. ${ }^{[16]}$ found abnormal changes in the morphology and structure of the vestibular system in an animal model of diabetes, mainly due to oxidative stress leading to an increase in lysosomes and fatty granules in ellipsoidal and balloon tissue. Another study ${ }^{[17]}$ found that diabetes causes degeneration and pathological alterations of vestibular type I hair cells in the microvascular end organs of diabetic rats, and hyperglycemia-induced metabolic stress can cause loss of type 1 hair cells in the balloon and cochlear nerve myelinolysis. In addition, vestibulocochlear nerve damage in diabetic rats has been demonstrated, including myelin lamina rupture and myelin thinning. ${ }^{[18]}$ In a clinical study, Li J et al. ${ }^{[19]}$ suggested that the blood supply to the vestibular end organs is a single terminal branch that is vulnerable to damageThe early stage of the disease manifests as peripheral vestibular impairment, which may be related to pathological and physiological alterations in nerve cells, axons and myelin sheaths due to increased sorbitol, fructose and decreased inositol. In this study, diabetic patients were grouped according to whether they had combined peripheral nerve damage, and patients in the SDPN and DDPN groups had vestibular nerve damage, which may be related to vestibular nerve demyelination injury.

The detection of diabetic vestibular function has received attention as the impact of vestibular impairment on balance and falls in diabetic patients has been gradually recognized.Different tests, such as Pure Tone Audiometry, video Head Impulse Test, Vestibular Evoked Myogenic Potential are used for the assessment of vestibular function. Ward et al. ${ }^{[20]}$ performed functional localization of vestibular damage in adults with type 2 diabetes and confirmed that both cVEMP interpeak amplitude and oVEMP $\mathrm{N} 1$ amplitude were reduced in the diabetic group. A study found that diabetic patients with severe DPN had more frequent vestibular dysfunction than diabetic patients without DPN (76\% vs $49 \%) .{ }^{[21]}$ kamali et al. ${ }^{[22]}$ found that cVEMP latency was significantly longer in type 1 diabetic patients with or without DPN, while amplitude values did not differ between study groups. In their study, prolonged cVEMP latencies were more common in the DPN group than in diabetic patients without DPN, and they speculated that this finding may indicate an association with damage to posterior vagal areas, particularly the vestibulospinal tract. In addition, diabetic patients may develop vestibular dysfunction in the absence of overt vestibular symptoms, called "subclinical vestibular neuropathy". In such cases, vestibular lesions can be identified by objective vestibular diagnostic testing tools, despite the absence of vestibular symptoms in patients with DM. oVEMP and cVEMP results were evaluated by Konukeven et al. ${ }^{[23]}$ in 30 diabetic patients, 30 prediabetic patients and 31 healthy controls without any central or peripheral vestibular lesions. In their study, oVEMP and cVEMP latencies were significantly longer in diabetic patients than in prediabetic patients and healthy controls, but there were no differences in amplitudes between the groups. The prolonged latency may be due to post-vagal demyelinating lesions or polyneuropathy, which is common in type 2 diabetes. In the present study, compared with healthy controls and NDPN group, the latencies of $\mathrm{n} 10$ and $\mathrm{p} 15$ of oVEMP and P13 and N23 of cVEMP were prolonged in the DPN group, which is a 
significant difference and supports the above-mentioned idea of "subclinical vestibular neuropathy". VEMP seems to be more meaningful in the detection of subclinical lesions. Cochlear vestibular impairment is slow to develop, and patients adapt to reduced vestibular function through visual and proprioceptive compensation to maintain body balance, ${ }^{[24]}$ with insidious clinical symptoms. The damage to vestibular function caused by hyperglycemia is easily overlooked during diabetes treatment, delaying vestibular function recovery.

Current studies on the effects of diabetes duration and glycemic control on vestibular function are inconsistent. In a study by Agrawal et al. ${ }^{[25]}$, it was concluded that longer duration of diabetes or higher glycosylated hemoglobin levels resulted in a higher incidence of vestibular dysfunction. Ward et al. ${ }^{[20]}$ concluded that duration of diabetes and glycemic control were not significantly correlated with vestibular impairment. In this study, we found that the duration of diabetes and glycemic control may correlate with vestibular damage, and the latency period was prolonged with increased glycosylated hemoglobin and fasting glucose levels, suggesting that poor glycemic control exacerbates vestibular impairment. The sample size of this study was relatively small, and further expansion of the sample size is needed for validation.

In conclusion, there is an increasing number of studies that consider vestibular function impairment as an important complication of diabetes mellitus, leading to balance dysfunction in diabetic patients and seriously affecting the quality of life. ${ }^{[26,27]}$ By studying the characteristics of vestibular function changes in diabetic patients, it is beneficial to increase the understanding of diabetic vestibular impairment, provide a basis for early detection and rehabilitation of diabetic vestibular impairment, reduce the incidence of falls in diabetic patients, and improve the quality of life.

\section{Declarations}

Data availability/Availability of Data and Materials

All data generated or analysed during this study are included in this published article [and its related files].

Ethics approval and consent to participate

This research was approved by the Medical Research Ethics Committee of Second Affiliated Hospital of Fujian Medical University, Quanzhou, P. R. China. All participants gave written consent after being informed about the procedure.

Consent for publication

All authors have reviewed the fnal version of the manuscript and agree with the publication of the information presented here.

Competing interests 
No potential conficts of interest relevant to this article were reported.

Funding

This study was supported by the startup fund for scientific research, Fujian Medical University (2020QH1122) .

Authors' contributions

Jiayu Lin conceived this project and supervised the experiments. Huapin Huang supervised the experiments. Jinying Zhang performed the experiments囚analyzed the data and wrote the paper.

Acknowledgements

This study was supported from the Second Affiliated Hospital of Fujian Medical University. We thank all of the participants in the study for their cooperation in this study.

\section{References}

[1]Ferber-Viart C, Dubreuil C, Duclaux R. Vestibular evoked myogenic potentials in humans: a review[J]. Acta otolaryngology,1999, 119(1): 6-15.

[2]Lagani V, Koumakis L, Chiarugi F, et al. A systematic review of predictive risk models for diabetes complications based on large scale clinical studies[J]. J Diabetes Complications, 2013, 27(4): 407-413.

[3] Yang W, Lu J, Weng J, et al. Prevalence of diabetes among men and women in China[J]. N Engl J Med, 2010, 362(12): 1090-1101.

[4]Agrawal Y, Carey J P, Della S C, et al. Disorders of balance and vestibular function in US adults: data from the National Health and Nutrition Examination Survey, 2001-2004[J]. Arch Intern Med,2013, 11(03):397-400.

[5]Konukseven Ozlem,Polat Sefika Burcak,Karahan Sevilay,et al.Electrophysiologic vestibular evaluation in type 2 diabetic and prediabetic patients: Air conduction ocular and cervical vestibular evoked myogenic potentials[J].Int J Audiol, 2015, 54: 536-43.

[6]Cohen Atsmoni S, Brener A, Roth Y. Diabetes in the practice of otolaryngology. Diabetes \& metabolic syndrome. 2019, 13(2): 1141-50.

[7]Murofushi T. Clinical application of vestibular evoked myogenic potential (VEMP). Auris Nasus Larynx. 2016 Aug;43(4):367-76.

[8]American Diabetes Association. Standards of medical care in diabetes-2012. Diabetes Care. 2012 Jan;35 Suppl 1(Suppl 1):S11-63. 
[9]American Diabetes Association. Diagnosis and classification of diabetes mellitus. Diabetes Care. 2010 Jan;33 Suppl 1(Suppl 1):S62-9.

[10]Liu B, Fu XX, Wu ZM, Zhou W, Zhu H. Expert consensus on clinical detection techniques for vestibular evoked myogenic potentials[J]. Chinese Journal of Otology,2019,17(06):988-992.

[11]Zhang S, Liu XX, Huang S, Fan DS. Establishing a detection method for vestibular evoked myogenic potentials and exploring its clinical significance[J]. Chinese Journal of Medicine,2018, 98(47):3868-3872.

[12]Khan S, Chang R. Anatomy of the vestibular system: a review. NeuroRehabilitation. 2013, 32(3): 43743.

[13]Gioacchini FM, Albera R, Re M, Scarpa A, Cassandro C, Cassandro E. Hyperglycemia and diabetes mellitus are related to vestibular organs dysfunction: truth or suggestion? A literature review. Acta Diabetol. 2018 Dec;55(12):1201-1207.

[14]Perez R, Ziv E, Freeman S, Sichel JY, Sohmer H. Vestibular end-organ impairment in an animal model of type 2 diabetes mellitus. Laryngoscope. 2001 Jan;111(1):110-3.

[15]Walley M, Anderson E, Pippen MW, Maitland G. Dizziness and Loss of Balance in Individuals With Diabetes: Relative Contribution of Vestibular Versus Somatosensory Dysfunction. Clinical diabetes: a publication of the American Diabetes Association. 2014;32(2):76-7.

[16]Myers S F,Ross M D.Morphological evidence of vestibular pathology in long-term experimental diabetes mellitus. II. Connective tissue and neuroepithelial pathology[J].Acta Otolaryngol, 1987, 104: 40-9.

[17]Bayram A. Vestibular evoked myogenic potentials in patients with diabetes mellitus. Journal of otology. 2019;14(3):89-93.

[18]Myers S F.Myelin-sheath abnormalities in the vestibular nerves of chronically diabetic rats[J]. Otolaryngol Head Neck Surg, 1998, 119: 432-8.

[19]Li Z, Zhang T Y, Shen J C, et al. Changes in vestibular function and its clinical significance in diabetic patients[J]. Journal of Clinical Otolaryngology-Head and Neck Surgery,2008,22(1):10-13.

[20]Ward Bryan K,Wenzel Angela,Kalyani Rita R, et al.Characterization of Vestibulopathy in Individuals with Type 2 Diabetes Mellitus[J].Otolaryngol Head Neck Surg, 2015, 153: 112-8.

[21]Chang Chih-Ming,Young Yi-Ho,Cheng Po-Wen.Age-related changes in ocular vestibular-evoked myogenic potentials via galvanic vestibular stimulation and bone-conducted vibration modes[J]. Acta Otolaryngol, 2012, 132: 1295-300.

[22]Kamali Behnoush,Hajiabolhassan Fahimeh,Fatahi Jamileh,et al.Effects of diabetes mellitus type I with or without neuropathy on vestibular evoked myogenic potentials[J].Acta Med Iran, 2013, 51: 107-12. 
[23]Konukseven Ozlem,Polat Sefika Burcak,Karahan Sevilay,et al.Electrophysiologic vestibular evaluation in type 2 diabetic and prediabetic patients: Air conduction ocular and cervical estibular evoked myogenic potentials[J].Int J Audiol, 2015, 54: 536-43.

[24] Huang X, Liu B, Sun J, et al. [Analysis and evaluation of the balance function in patients with type 2 diabetes][J]. Lin Chung Er Bi Yan Hou Tou Jing Wai Ke Za Zhi,2016,30(1):27-30.

[25]Agrawal Y, Carey J P, Della S C, et al. Diabetes, vestibular dysfunction, and falls: analyses from the National Health and Nutrition Examination Survey[J]. Otol Neurotol, 2010, 31(9): 1445-1450.

[26]Zidong Jiang, Fan Gao, Junhua Hou, Clinical analysis of intractable vertigo syndrome, Chinese Journal of Otology, 2012(02):155-159.

[27] Zhou Y, Wu ZH, Hou JN. Quality of life in 1888 vertigo patients. Chinese Journal of Otology, 2010, 8(04): 434-436.

\section{Supplementary Files}

This is a list of supplementary files associated with this preprint. Click to download.

- rawdata.xIsx 